\title{
Damage detection by recurrence and entropy methods on the basis of time series measured during composite milling
}

\author{
Krzysztof Kecik $^{1} \cdot$ Krzysztof Ciecielag $^{2} \cdot$ Kazimierz Zaleski $^{2}$
}

Received: 27 May 2020 / Accepted: 1 September 2020 / Published online: 30 September 2020

(C) The Author(s) 2020

\begin{abstract}
This paper presents methods for damage detection in machined material on the basis of time series measured during milling of glass-fiber-reinforced polymer (GFRP). Recurrence methods and different types of entropy have emerged as useful tools for detecting subtle non-stationarities and/or changes in nonlinear signals. In this research, a recurrence plot, recurrence quantifications, an approximate entropy, and sample entropy are used. By identifying changes in the cutting force measured during the composite milling process, the damage occurrence has been detected. Firstly, the damage has been modelled as the intentionally introduced hole with different diameters and depths in order to estimate the size detectable damages and to select proper recurrence measures as damage indicators. Next, the experiments with the real damage have been performed and the damage indicators have used.
\end{abstract}

Keywords Damage detection $\cdot$ Recurrence $\cdot$ Entropy $\cdot$ Nonlinear time series

\section{Introduction}

\subsection{Composite defects and their detection}

The machinability problem of steel (hard materials) and composite materials is becoming more and more important because production times are dramatically reduced due to the much higher cutting parameters. The machinability can be difficult to predict because machining has so many variables. Generally, the machinability has been mainly assessed based on tool wear (tool life), cutting forces, power consumption, and surface finish. The prediction of cutting forces during micro-milling based on the chip thickness accumulation phenomenon is presented in [1]. The authors proposed a force model including the micro-end milling kinematics, geometric errors of the machine tooltoolholder-mill system, elastic and plastic deformations of workpiece correlated with the minimum uncut chip

Krzysztof Kecik

k.kecik@pollub.pl

1 Department of Applied Mechanics, Lublin University of Technology, Lublin, Poland

2 Department of Production Engineering, Lublin University of Technology, Lublin, Poland thickness, and flexibility of the slender micro-end mill. Moreover, their study includes the instantaneous area of cut. This team's next paper [2] presents analysis on metrological relations between instant tool displacements and surface roughness during precise ball end milling. The new method describing the working part vibration of ball end mills is proposed. The prediction of the surface topography and optimization of the cutting parameters for obtaining desired surface roughness are shown in [3]. Grossi et al. [4] described milled surfaces under machining vibration (chatter). Analytical formulations to compute surface quality are proposed.

Machining composites is different from machining metals due to interaction with the cutting tool and is a complex problem. Generally, composites are associated with two or more different material components. Usually, the composites have better performance from any of the initial ones. The common set is the dispersion of the reinforcement in a matrix which is stronger than the matrix. However, the higher properties of a composites occur when they are properly designed. The most used composite materials are reinforced with fibers and therefore can be highly anisotropic. The fiber component is very important and is usually responsible for the improvement of the matrix, and can be obtained via a wide range of methods, including wet layup, spray-up, preimpregnated (prepreg) layup, and others. 
The damages or defects in the composite materials can be inadvertently produced during the manufacturing process or in the course of normal use. The manufacturing process and normal use cause a wide range of imperfections. They can be located in an external or internal structure. The external damages especially large (macroscopic) can be visually inspected, such as dimensions, finish, and warpage. The most important defects are deaminations (separations within plies of a laminate), inclusions (foreign matter embedded), porosity and voids (entrapped air and gas bubbles), impact damage, fiber misalignment (distortion of the plies), fiber wrinkling, fiber buckling, fiber breakage, core crushing, etc. $[5,6]$.

In general, we have two basic methods for the detection of defects and damages: destructive and non-destructive. The main disadvantage of destructive method is that the tested materials cannot be further used. Nevertheless, to derive the size and position of defects, these methods are widely used [7]. Tensile, bending, and impact testing are some examples of destructive methods. The nondestructive testing is a solution to assure safety without destroying the components. Visual, ultrasonic, thermography, radiographic, electromagnetic, and acousto-ultrasonic are some common non-destructive methods. One of the most used non-destructive methods is ultrasonic inspection from which a two-dimensional map is received [8]. Ultrasound pulses are reflected by interfaces between materials of different properties. In the case of defects, this can cause a discrete reflection or transmission loss [9]. The most commonly test is the C-scan map of the structure [10]. Generally, ultrasound methods are very sensitive to most types of defects commonly found in composites. However, these methods are useful if the response on a defective structure must be significantly different from that on a sound structure. One of the newer methods is laser ultrasound generation, but the cost of its use is high. The radiography inspection like neutron radiography (NR) or X-ray radiography (XR) is used for detecting the fiber breaking, resin-rich, or starved areas [11]. Optical and thermal methods are limited by the depth of penetration and more expensive than classic ultrasound methods. Another method is the lowfrequency vibration test. This method for the sandwich structures [12-14] is usually applied.

\subsection{Nonlinear time series method}

All earlier mentioned methods are based on the structural analysis and ineffective when defects arise during the manufacturing process. The different kinds of surface and structural defects or damages may appear during the machining process. Therefore, the method which would allow detecting damages directly during the manufacturing process would be very helpful and useful.
The interesting advanced technique is the nonlinear time series analysis (NTSA). This was primarily because most experimental time series were one-dimensional scalar measurements. The millstone of the NTSA were papers of Takens and Packard $[15,16]$, which reconstruct the full dynamics of a complicated nonlinear system from a single time series. The reconstruction, if properly done, can be extremely useful because it guarantees to be topologically identical to the full dynamics. Nonlinear concepts began to be increasingly applied to complex systems from different fields. The well-known NTSA methods are as follows: Lyapunov exponent, information dimension, correlation dimension, entropy, Poincare map [17-19], and recurrence plots. Most of these methods are based on investigating the evolution of trajectories in the phase space. The NTSA can be applied in different engineering aspects; one of the most promising is structural health monitoring (SHM) [20]. This is possible because the damage changes the material properties. The SHM method is based on the observation of a system response from sensors and is trying to catch damage-sensitive features which are statistically analyzed.

One of the most interesting NTSA method is the recurrence plot (RP) method which has greater sensitivity to changing dynamics than other methods. RP is the graphical representation of the recurrence states of a dynamical system. The statistical analyses of the recurrence structure are collectively referred as recurrence quantification analysis (RQA) and they form the core of RP techniques. A crucial advantage of RP techniques over other nonlinear methods is that they perform reasonably well even when the length of the nonlinear time series is very short (even 50-100 data points) and in cases when the underlying system is not sufficiently deterministic have proven useful in characterizing their behavior [21]. Therefore, the RP and RQA methods may be used in all types of signals including periodic, non-stationary, and chaotic [22].

The recurrence methods are very popular in different engineering branches. One of the uses is the monitoring of chatter detection during the machining process [23]. The authors analyzed the nonlinear time series obtained with increasing speed of milling process. They noticed that the RQA parameters (particulary determinism) could be used to improve diagnostics and invent a chatter feedbackcontrol procedure in the milling operation. Kecik et al. $[24,25]$ have studied the stability of a high-speed milling process of nickel superalloys. Their results show that the best parameters to classify the kind of motion and find the stability border are the divergence and the longest line.

Recurrence methodologies have also been used in damage detection and damage analysis. Iwaniec et al. [26] use the RQA method to analyze the change in dynamics between cracked and uncracked metal and aluminum plates. Nichols et al. [27] used multivariate RQA techniques to 
dynamical change analysis induced by a cut on the plate. A recurrence-based entropy to construct a data-driven model of ball bearing degradation is analyzed by Qian et al. [28]. Investigation of the corrosion of metal surfaces by RP and RQA methods to distinguish different types of corrosion dynamics is presented in papers $[29,30]$. They showed that the RQA parameters can be used for corrosion detection.

\subsection{Motivation}

Inspired by many successful applications of the RP and RQA methods, this paper is intended to make use of recurrence methodology and study damage detection basis on the milling forces. This paper is a continuation and significant extension with new elements of our paper [31], where we have studied the possibility of damage detection. Some of the promising recurrence quantifications as damage indicators have been selected. However, in previous our paper, we analyzed the influence of only the damage diameter.

In this paper, we expanded our research and we analyze both the diameter and depth of the damage. Moreover, two new methods based on entropy analysis have been added. The goal here is to extend the use of RP, RQA, and entropy to detect damage in a composite structure.

\section{Recurrence and entropy analysis}

\subsection{Phase space reconstruction}

The analysis of phase space trajectories is a basic concept of nonlinear data analysis. Recurrence plot (or recurrence diagram) is a graphical tool based on phase space reconstruction which begins with a time-delay embedding of the data. The dynamics of the nonlinear system can be described by measured data consisted of one time series. This means that the phase space of a dynamical system can not be obtained from the measurement directly. The literature studies prove that it is possible to reconstruct the entire dynamics of a system from a relatively small number of analyzed data [32]. This is because the different degrees of freedom of a dynamic system interact with each other; the combination of all other components is concealed in each observable quantity through the main state vector components. Takens' theorem $[15,18]$ allows the formation of $N$-dimensional vector $X(i)$ from the nonlinear time series. Assuming that the measured data are $x(1), x(2), x(3), \ldots, x(n)$, the reconstructed vector $X(i)$ is defined as:

$$
\begin{aligned}
X(i) & =\{x(i), x(i+d), \ldots, x(i+(m-1) d)\}, \\
i & =1,2, \ldots, N-(m-1) d,
\end{aligned}
$$

where $m$ and $d$ are called embedding dimension and embedding delay, and $i$ is a point in the reconstructed $m$-dimension space. Embedding parameters $d$ and $m$ are crucial to reveal the underlying dynamics of a complex system. The most often used method is the Average Mutual Information Function (AMI) for computing $d$ [33], and the False Nearest Neighbors (FNN) method for $m$ [34]. One chooses the first minimum location of the AMI function as $d$. Second, the percentage of FNN function is calculated for each $m$ value. The parameter $m$ is assumed to be found for the first percentage of FNN function dropping close to zero. Both methods are described in papers $[33,34]$ and in our paper [31].

\subsection{Recurrence plot}

Based on the measured time series from the real process, the reconstructed vector can be obtained from Eq. 1 . Introducing the threshold $\epsilon$ then a two-dimensional matrix can be obtained by comparing the distance between the vectors $[35,36,38,51]$ :

$$
R P_{i, j}(m, \epsilon)=H(\epsilon-\|X(i)-X(j)\|), i, j \in N,
$$

where:

$\epsilon$ is the threshold,

$\|\cdot\|$ is a norm (usually Euclidean or maximal),

$H(\cdot)$ is the Heaviside function.

$\epsilon$ depends on dynamic changes, representing the specific length scale of focus. Usually, it is relative to the standard deviation [39]. The norm parameter determines the size and shape of the neighborhood surrounding each reference point. The distance between the $X(i)$ and $X(j)$ states defines whether each state is recurrent or not. If the distance is smaller then $\epsilon$ at the time a black point is scored. After all vectors are processed, the recurrence diagram is drawn. The RP plot consists of single dots and diagonal lines, as well as vertical and horizontal lines, and even bowed lines. If the analyzed signal is deterministic, then the RP shows lines parallel to the main diagonal.

\subsection{Recurrence quantification analysis}

The recurrence diagram is a rich qualitative description of the nonlinear dynamics. However, the quantitative analysis is difficult and requires more advanced techniques. Zbilut and Webber [36, 37] described statistics of the RP plot based on the line structures and point density. They introduced a series of recurrence parameters: recurrence rate $(R R)$, determinism $(D E T)$, and entropy $(E N T)$. The RP method was further extended by Marwan et al. [40] with the introduction of additional quantifications, such as laminarity ( $L A M)$ and the trapping time (TT). In 2000, Gao and Cai [41] introduced recurrence times of the first $(T 1)$ and second (T2) types. Moreover, Marwan et al. [42] propose the clustering coefficient $(C C)$ and the transitivity $(T R A N S)$. 
Mathematically, these indicators are calculated based on equations listed in Table 1. $R R, D E T, E N T$, and $L_{\max }$ variables are based largely on the lengths, number, and distributions of diagonal lines. Physically, $R R$ means the density of recurrence points in the RP plot. Determinism is the ratio of recurrence points that form the diagonal structure to all recurrence points. DET characterizes the predictability of a time series. Entropy is the Shannon entropy of the probability distribution of the diagonal lines. This parameter can be used to estimate the complexity of the dynamics system. The information about vertical lines can be analyzed by $L A M, T T$, and $V_{\max }$. Laminarity reflects vertical structure points and indicates the laminar phases. $T T$ contains information about the amount and the length of the vertical structures, and $V_{\max }$ measures the longest vertical line.

The total procedure of the RP and RQA in Fig. 1 is shown. The measured nonlinear signal is normalized. Each time series was subjected to the process of normalization by a common method: normalizes the signal to mean $(-)$ and standard deviation $(\sigma)$.

Next, the embedding parameters ( $d$ and $m$ ) and threshold $(\epsilon)$ have been calculated/estimated and the phase space has been reconstructed. RP and RQA methods can be used for all types of nonlinear signals.

\subsection{Entropy-based complexity measures}

The entropy has emerged as an appropriate complexity measure in the study of nonlinear time series in many fields of science, information theory, statistical mechanics, chaos theory, and neural networks [43]. The change of entropy is inversely related to the change of free energy. In the literature, we can meet different types of entropy: Shannon, minimum and maximum, Renyi, sample, spectral, approximate, and others [44]. Two commonly used methods are the approximate entropy (ApEn) and the sample entropy (SampEn) providing a statistic to assess complexity from the noisy short-length data [45]. ApEn entropy was developed by Pincus [46] as a measure of regularity to quantify levels of complexity within a time series.

The approximate entropy is defined as:

$\operatorname{ApEn}(m, \epsilon, N)=\phi^{m}(\epsilon)-\phi^{m+1}(\epsilon)$,

where the probability of pattern of length $\phi^{m}(\epsilon)$ and the correlation sum $C_{i}^{m}(\epsilon)$ are estimated from:

$$
\begin{gathered}
\phi^{m}(\epsilon)=\frac{1}{N-(m-1) d} \sum_{i=1}^{N-(m-1) d} \ln C_{i}^{m}(\epsilon), \\
C_{i}^{m}(\epsilon)=\frac{1}{N-(m-1) d} \sum_{j}^{N-(m-1) d} H\left(\left|X_{i}, X_{j}\right|-\epsilon\right) .
\end{gathered}
$$

\begin{tabular}{|c|c|c|}
\hline Quantification & Equation & Description \\
\hline Recurrence rate $(R R)$ & $\frac{1}{N^{2}} \sum_{i, j=1}^{N} R P_{i, j}(m, \epsilon)$ & Percentage of darkened points \\
\hline Determinism $(D E T)$ & $\frac{\sum_{l=l_{\min }}^{N} l P(l)}{\sum_{i, j=1}^{N} R P_{i, j}(m, \epsilon)}$ & Percentage of points forming diagonal lines \\
\hline Entropy $(E N T)$ & $-\sum_{l=l_{\min }}^{N} P(l) \ln (P(l))$ & Entropy of the probability distribution of diagonal lines \\
\hline Laminarity $(L A M)$ & $\frac{\sum_{v=v_{\min }}^{N} v P(v)}{\sum_{v=1}^{N} v P(v)}$ & Fraction of recurrence points forming vertical lines \\
\hline Trapping time $(T T)$ & $\frac{\sum_{v=v_{\min }}^{N} v P(v)}{\sum_{v=v_{\min }}^{N} P(v)}$ & Average length of vertical lines \\
\hline Longest diagonal line $\left(L_{\max }\right)$ & $\max \left(\left\{l_{i} ; i=1, \ldots, N_{l}\right\}\right.$ & Length of the longest diagonal line \\
\hline Longest vertical line $\left(V_{\max }\right)$ & $\max \left(\left\{v_{i} ; i=1, \ldots, N_{v}\right\}\right.$ & Length of the longest vertical line \\
\hline Averaged diagonal line $(L)$ & $\frac{\sum_{l=l_{\min }}^{N} l P(l)}{\sum_{l=l_{\min }}^{N} l P(l)}$ & Length of average diagonal line \\
\hline Recurrences time $(T 1)$ & $\left.\mid\left\{i, j: \overline{x_{i}}, \overline{x_{j}}\right\} \in R_{i}\right\} \mid$ & Time distances of recurrence points in vertical direction \\
\hline Recurrences time $(T 2)$ & $\left.\mid\left\{i, j: \overline{x_{i}}, \overline{x_{j}}\right\} \in R_{i}, \overline{x_{j}} \notin R_{i}\right\} \mid$ & Time distances of recurrence points in vertical direction \\
\hline Recurrence time entropy $(R T E)$ & $-\frac{1}{\ln V_{\max }} \sum_{v=1}^{V_{\max }} H_{v}(v) \ln H_{v}(v)$ & Measure quantifies the extent of recurrences \\
\hline Transitivity $(T R A N S)$ & $\frac{\sum_{i, j, k=1}^{N} R P_{i, j} R P_{j, k} R P_{k, i}}{\sum_{i, j, k=1}^{N} R P_{i, j} R P_{k, i}}$ & A quantitative measure of dynamical complexity \\
\hline
\end{tabular}

Table 1 Quantification of recurrence plots [36, 39, 41]

$P(l)$ and $P(v)$ represent the distribution of the lengths of diagonal and vertical lines, respectively. $N_{l}$ and $N_{v}$ are numbers of diagonal and vertical lines in the RP. $R_{i}$ are the recurrence points which belong to the state $\overline{x_{i}}$, the $H_{v}(v)$ is distribution 


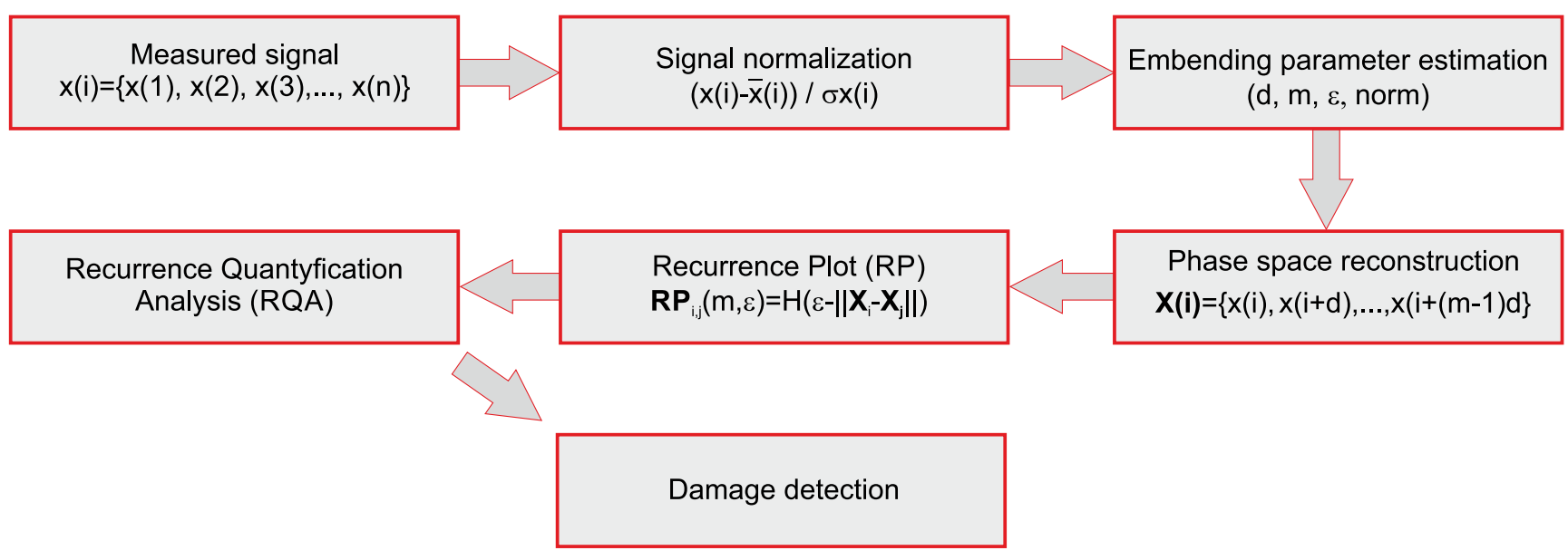

Fig. 1 The framework of recurrence analysis

The term $\left|X_{i}, X_{j}\right|$ is a measure of the maximum distance between $X_{i}$ and $X_{j}$. The computation of ApEn requires the selection of embedding parameters $m, d$, and threshold $\epsilon$ (like in phase space reconstruction). Generally, we can say that the ApEn measures the logarithmic likelihood that two points $\left(X_{i}, X_{j}\right)$ that are close in an $m$-dimensional space remain close in an $(m+1)$-dimensional space. The disadvantages of ApEn are that it is strongly dependent on the signal length and is often lower than expected for short records and additionally lacks relative consistency [47, 48].

To overcome the disadvantages of ApEn, the sample entropy was proposed [49] by Richman et al. In order to calculate the SampEn, the probability $\phi^{m}(\epsilon)$ and $\phi^{m+1}(\epsilon)$ that two sequences match for $m$ points is computed by counting the average number of vector pairs:

$\phi^{m}(\epsilon)=\frac{1}{N-m d} \sum_{i=1}^{N-m d} C_{i}^{m}(\epsilon)$.

Then, the SampEn can be calculated as:

$\operatorname{SampEn}(m, \epsilon, N)=-\ln \frac{\phi^{m}(\epsilon)}{\phi^{m+1}(\epsilon)}$.

Sample entropy is independent of the length of time series and usually displays relative consistency under various conditions [47].

\section{Experiment}

\subsection{Milling test}

The milling processes have been conducted on machining center Avia VMC800HS, equipped with Heidenhain control. The experiment was performed using Kennametal cutter with a diameter of $20 \mathrm{~mm}$ and symbol of
20A02R028A20ED10 (dedicated for composites). The cutter has indexable inserts covered by polycrystalline diamond (manufacturer's grade KD1410). The insert parameters were as follows: insert included angle $75^{\circ}$, clearance angle $15^{\circ}$, rake angle $0^{\circ}$, and corner radius $0.4 \mathrm{~mm}$. The insert sizes are as follows: thickness $3.75 \mathrm{~mm}$, width $6.75 \mathrm{~mm}$, and length $12.04 \mathrm{~mm}$. In the research, the end milling of slots was applied.

The cutting speed was set to $250 \mathrm{~m} / \mathrm{min}$ (high-speed machining) and the cutting depth $a_{p}=2 \mathrm{~mm}$, while the feeding rate $0.2 \mathrm{~mm} / \mathrm{blade}$. The machining process was done under dry condition. Vibration signals were acquired using 3D Kistler dynamometer (type 9257B) connected to a dedicated charge amplifier (Kistler 5070). The Dynoware data acquisition card (5697A) with the Dynowave software was used for data acquisition. Data was acquired at a sampling frequency of $10 \mathrm{kHz}$. Moreover, no signal filtration/processing was applied. The milling center photo and scheme of the measuring system in Fig. $2 a$ and $b$ are shown.

The machining material was a glass-fiber-reinforced polymer with epoxy resin. This type of material was chosen due to its wide application in the aviation industry (e.g., the aircraft stabilizers or slats). The main reasons for GFRP application are the high stiffness of the fibers, non-corrosivity, high tensile strength, lightweight, and high strength to weight ratios.

The GFRP workpiece material has a thickness of $15 \mathrm{~mm}$ and consists of fifty prepreg sheets oriented in configuration $0^{\circ} \div 90^{\circ}$. The temperature, humidity, and the amount of impurities in the controlled room were maintained at a preset level, namely at temperature range of $18 \div 30{ }^{\circ} \mathrm{C}$, humidity up to $60 \%$, and the amount of impurities not exceeding $10^{4}$ particles per $1 \mathrm{~m}^{3}$. All milling tests were carried out in one clamping, which ensured similar conditions (i.e., stiffness, damping). 
Fig. 2 Photo of milling test (a) and scheme of the measuring system; 3D dynamometer, charge amplifier, data acquisition module. and $\mathrm{PC}$ with the Dynowave software (b) (a)

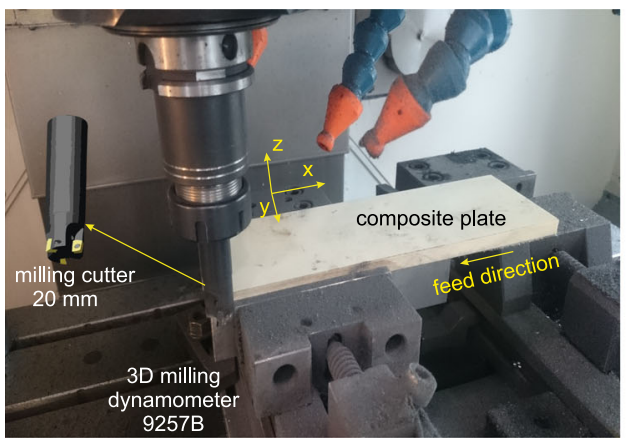

(b)

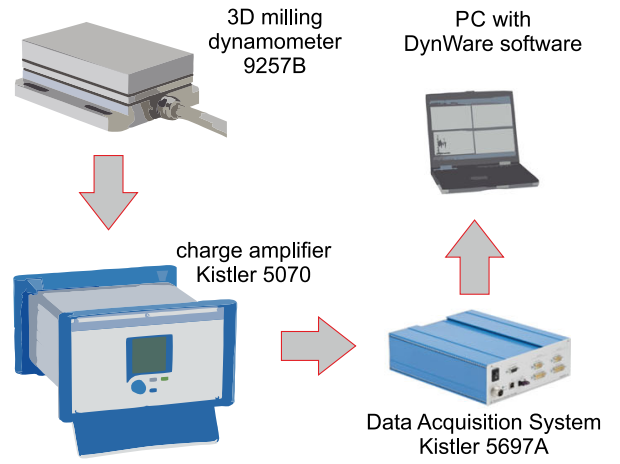

\subsection{Damage modelling}

The purpose of this paper is to evaluate the size of the detectable damages from the nonlinear time series of milling forces. The holes of different diameters and depths have been drilled to simulate discrete real damages in GFRP material. The scheme of machining of composite plate with the intentionally introduced damages in Fig. 3 is shown. The damages were introduced in two forms. Firstly, the damages (through-holes) with different diameters of $0.8 \mathrm{~mm}, 1 \mathrm{~mm}$, $1.2 \mathrm{~mm}$, and $1.5 \mathrm{~mm}$ were drilled (test no. 1). The second test (no. 2) was based on the holes with constant diameter ( $2 \mathrm{~mm}$ ) and different depths of $0.5 \mathrm{~mm}, 1.0 \mathrm{~mm}, 1.5 \mathrm{~mm}$, and $2.0 \mathrm{~mm}$.

In order to conduct RP and RQA analyses, the cutting forces in the feed direction ( $x$ direction, see Fig. 2a) have been measured. We analyze the different experimental signals; therefore, the normalizations of the signal to mean and standard deviation have been applied [39]. The detailed description of this procedure can be found in paper [31]. Figure $4 \mathrm{a}$ and $\mathrm{b}$ show the feed cutting force measured from test nos. 1 and 2.

The blue color means the real milling force signal measured by the dynamometer $\left(F_{x}\right)$; the red color is the milling signal after normalization $(F)$. The normalization reduces the problem of a vibration center shift. The dashed lines show position of the damages (holes). Upon analyzing, it can be seen that for test no. 1 (Fig. 4a) the cutting force in the damage position is increased, and the differences are visually observed for all damage diameters. The increase of milling force is due to disorder at the structural milling signal caused by holes (provably the cutter blades hit the hole). However, for damage with different depths (test no. 2, Fig. 4b), the milling force is increased only for depths

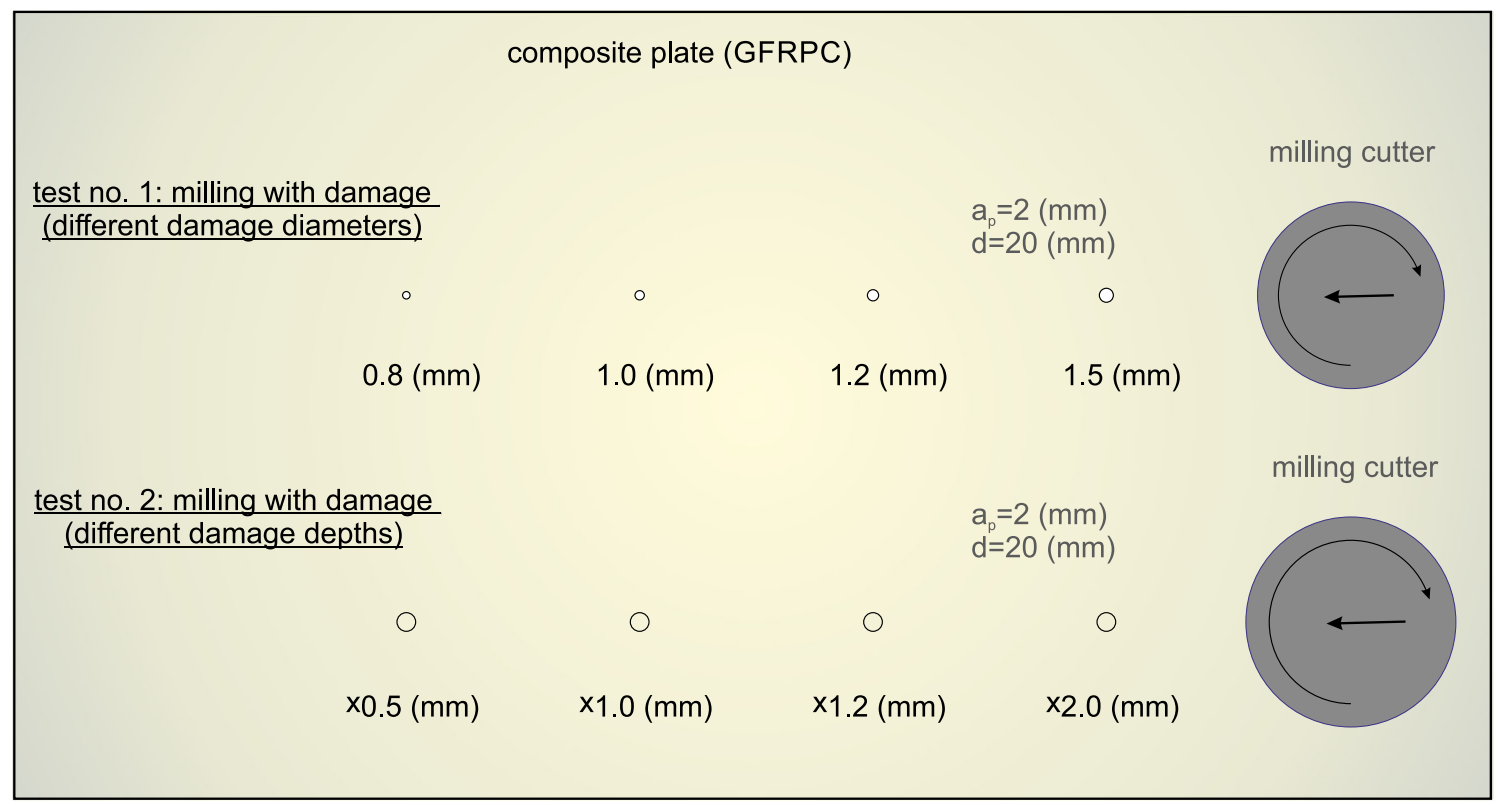

Fig. 3 Scheme of milling process with the intentionally introduced damages. The first test was with the different damage diameters; the second test with the different damage depth. The cutter diameter and the cutting depth were assumed $d=20 \mathrm{~mm}$ and $a_{p}=2 \mathrm{~mm}$ 
(a)

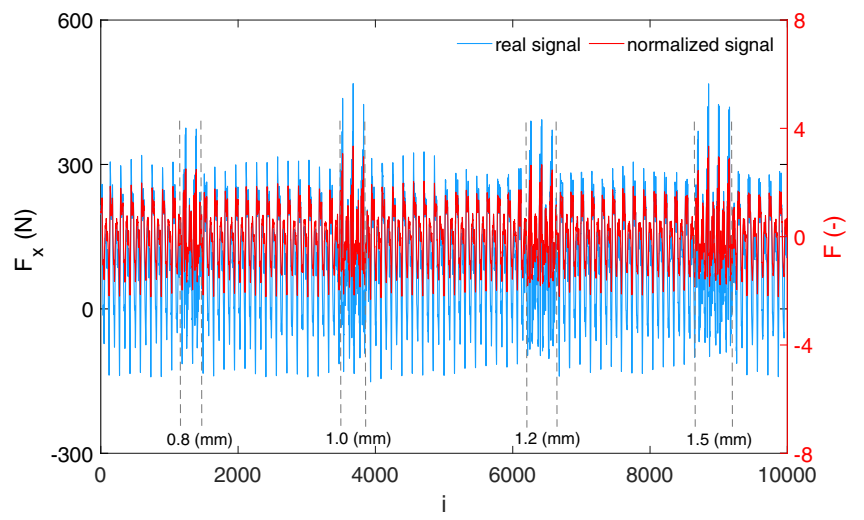

(b)

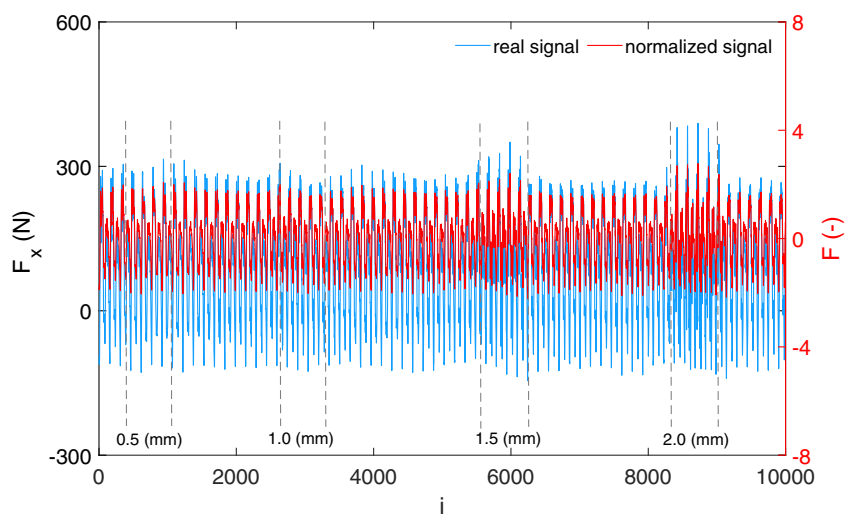

Fig. 4 Experimental measured cutting forces from milling test no. 1 (a) and no. 2 (b). The blue color means direct milling force, while the red color shows normalized cutting forces. The amplitude force jumps show the damage location (dashed lines). As we can see, only the higher damages are clearly visible

$1.5 \mathrm{~mm}$ and $2 \mathrm{~mm}$. This means that damage depth is very important and its detection from cutting force signal needs more advanced methods.

\section{Results and discussion}

\subsection{Recurrence diagram analysis}

Based on the cutting signals, the phase space is reconstructed and the recurrence plots are drawn. The first steps were estimation of embedding dimension and embedding delay using AMI and FNN methods. These parameters for both tests are the same. A lot of methods could be used to select the threshold, which strongly influences the RP diagram and RQA results. We assumed a common approach and assumed this as 0.2 of the standard deviation. The RP diagrams of test no. 1 (different hole diameters) and test no. 2 (different hole depths) in Fig. 5a and b are shown.

From both pictures, it can be seen that the density of recurrence points and vertical or horizontal lines in the RP plots are similar. The RP is mainly composed of long diagonal lines and empty spaces. The diagonal lines indicate the periodic behavior, while the empty spaces correspond to the damage positions. Moreover, the vertical and/or horizontal lines represent time segments that remain unchanged or change very slowly, and they are typical behaviors of the state of the laminar. Note that we evidently observe the empty spaces for all different holes (Fig. 5a). This means that all the damage diameters are clearly visible in the recurrence structure, and all damages can be easily detected by recurrence methods. However, the RP diagram obtained for the different damage depth reveals that the biggest damages are visible $(1.5 \mathrm{~mm}$ and $2 \mathrm{~mm})$. This suggests that the damage smaller than $1.5 \mathrm{~mm}$ is rather difficult to detect directly from the RP plot and needs more advanced recurrence analysis. The RP and RQA analyses have been performed using CRP Toolbox for Matlab [50].

\subsection{Recurrence quantitative analysis}

The recurrence plots exhibit different structures which can be quantitatively described by recurrence quantifications (measures). One of the more effective RQA technique is the "moving windows" which yield time-dependent RQA measures [51]. The moving window of size $\delta$ shifted with a step $\delta i$ over the studied time series. Finally, we obtain time-dependent RQA results in the form of a periodogram. Of course, the "moving windows" analysis uses the same embedding dimension, delay, and threshold as before.

The statistics of $R R, D E T, L A M, E N T, T T, L, L_{\max }$, $V_{\max }, T T, T_{1}, T_{2}, R T E$, and $T R A N S$ for test no. 1 and no. 2 are shown in Fig. 6 (for different damage diameters) and Fig. 7 (for different damage depths).

As has been shown, the statistical parameters of the RQA analysis effectively detects dynamical changes in the signals. Analysis of Fig. 6 indicates sudden changes in the cutting force at the damage positions. This means that damages will lead to a perturbation of the signal, which can be observed by the RQA analysis. For example, the $R R$ quantification is on the level of 0.01 (constant value), but in the damages positions drop significantly to about 0.001 (ten times) (Fig. 6a). Similarly, other indicators significantly decreased their value. This indicates that practically all recurrence measures could be used as damage indicators. However, the most significant difference is observable for the following: $R R$ (Fig. 6a), DET (Fig. 6b), ENT (Fig. 6c), $L A M$ (Fig. 6d), $V_{M A X}$ (Fig. 6f), $T 1$ (Fig. 6g), $T 2$ (Fig. 6h), RTE (Fig. 6k), and T RANS (Fig. 61). All results obtained for the different diameters show that all holes can 
Fig. 5 Recurrence plots obtained from test no. 1 (a) and no. 2 (b). The upper figures show normalized cutting force signals applied for recurrence analysis. Note that RP diagrams are symmetric; therefore, both axes have the same meaning (horizontal and vertical lines). The empty spaces mean the damage location. The RP matrixes obtained for $m=5$, $d=17$, and $\epsilon=0.2$ of the standard deviation of the amplitude values (a)

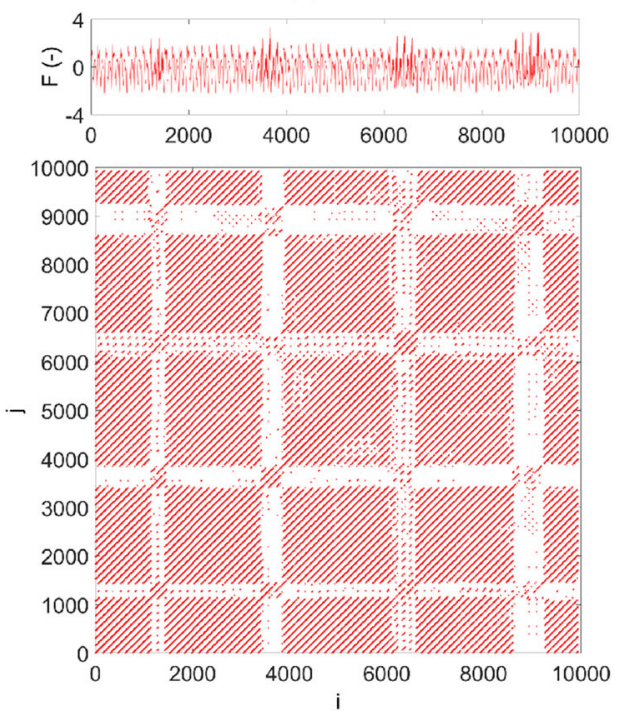

(b)

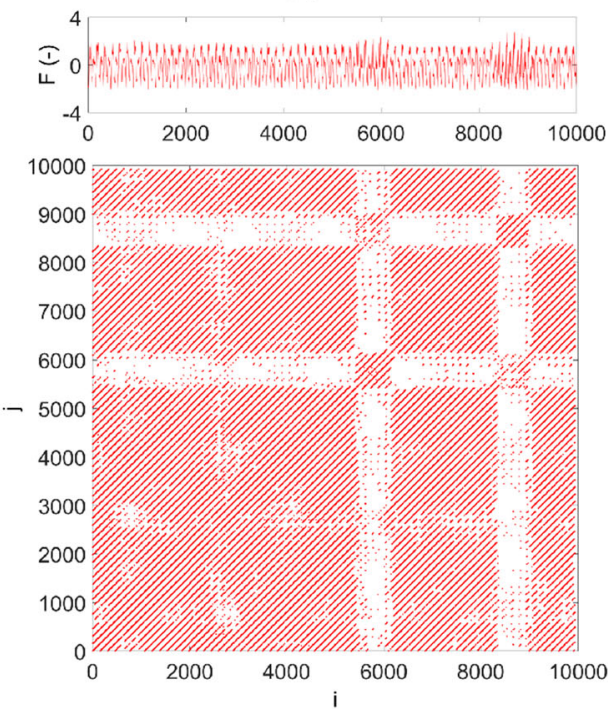

be detected by RQA methods. Referring it to the crosssectional area, we can indicate that we are able to detect the damage which is at least $4 \%$ of the cross-section area of the cutter. Smaller damages (holes) could not be tested due to technological reasons.

However, for the damages with different depths (and constant diameters of $2 \mathrm{~mm}$ ), the situation is changed (Fig. 7). The damage about the smallest depth $(0.5 \mathrm{~mm})$ is rather very difficult to detect. Only at the least depth of $1 \mathrm{~mm}$ or more is it possible to detect. The damaged crosssection area possible to detect is about $5 \%$ of the crosssection area of the cutter. In this case, the best recurrence measures are the following: $R R$ (Fig. 7a), $D E T$ (Fig. 7b), $E N T$ (Fig. 7c), LAM (Fig. 7d), T1 (Fig. 7g), T2 (Fig. 7h), $R T E$ (Fig. 7k), and TRANS (Fig. 7l). Other recurrence indicators show too small changes to be used to detect damages ( $L_{M A X}$ in Fig.7e, $V_{M A X}$ in Fig.7f, $L$ in Fig.7i, and $T T$ in Fig.7j). Upon comparing both results (Figs. 6 and 7), we can conclude that the depth of damage is crucial; the recurrence measures decrease if the hole depth is smaller.

In order to estimate the RQA measure level for damage detection, the optimal values based on the critical values (red dashed lines in Figs. 6 and 7) have been proposed. Because the damage depth strongly influences detection, it was assumed that the optimal recurrence quantification consists of $70 \%$ damage depth and 30\% damage diameter. These optimal values are listed in Table 2, and will be used later for the real damage detection.

Concluding the above, it can be said that the RQA method can be successfully applied to damage detection from the time series. The recommended RQA measures are $R R, D E T, E N T, L A M, T 1, T 2, R T E$, and TRANS.

Two similar recurrence quantifications were selected in [27], where two of the metrics $R R$ and $D E T$ showed a greater sensitivity to damage. The $D E T$ was selected in the spindle speed control to eliminate the vibration chatter in [23]. The chatter vibration detection by $R R, D E T$, $L_{\max }, V_{\max }$, and $L A M$ is shown in paper [25]. However, compared with [26], the results are different, where $T T$, $V_{\max }$, and $L$ were the best RQA measures indicating changes in determinism and also parameters that could possibly distinguish between the undamaged and cracked plates. Note that in mentioned papers, not all recurrence quantifications were analyzed.

The proposed method was checked for other materials (carbon fiber-reinforced polymer) and different ranges of parameters in $\mathrm{PhD}$ thesis [52] and papers [53-55].

\subsection{Entropy analysis}

Entropy is a measure of complexity and randomness of a nonlinear signal, describing the rate of information creation. Therefore, it can be used for nonlinear time series. In Figs. 8a and b, ApEn (red line) and SampEn (blue line) entropy are shown. The analysis has been performed for the same embedding parameters as recurrence methodology: $m=5, d=17$, and $\epsilon=0.2$ of the standard deviation of the amplitude. Moreover, the same technique "moving window" was applied.

The SampEn entropy results show its ability to distinguish between different systems' dynamics in the damage positions. The value of SampEn for the damage increases time twice (to 0.5). Similarly, like recurrence methods, the SampEn entropy can detect all holes with different diameters (through-hole), and only damages with $1.5 \mathrm{~mm}$ and $2 \mathrm{~mm}$ depths. Both critical values of the SampEn are similar and equal ( 0.38 (different diameters) and 0.35 (different depths)). Using the same algorithm as for recurrence 
Fig. 6 Statistical results of RQA analysis obtained from recurrence diagram in Fig. 5a. The embedding parameters are $m=5$ and $d=17$, and the window sliding technique parameters were $\delta=300$, $\delta i=1$. The depth of cut was $a_{p}=2 \mathrm{~mm}$. Changes in the RQA values can be used as the damage indicators (a)

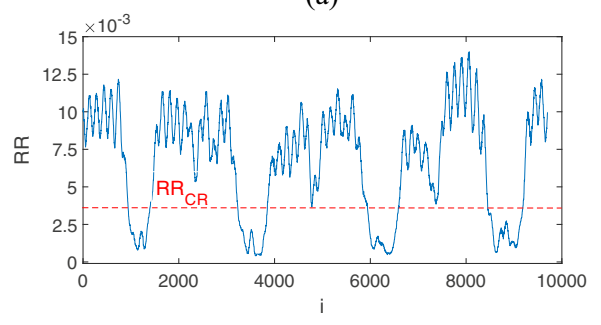

(c)

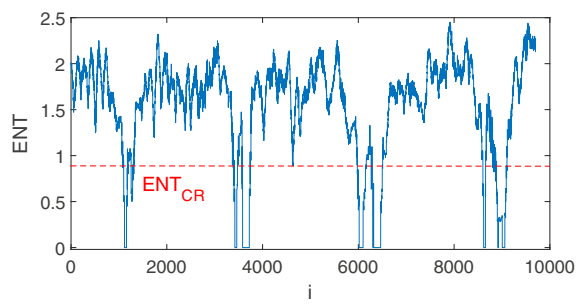

(e)

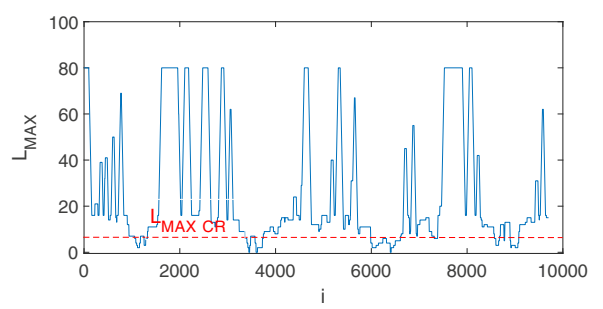

(g)

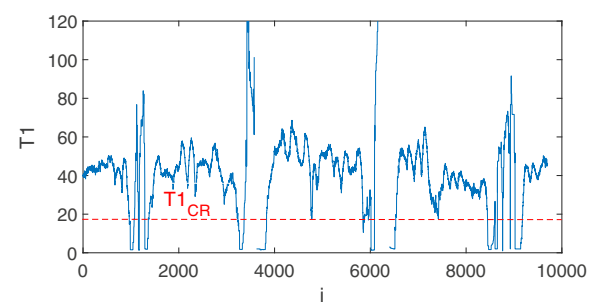

(i)

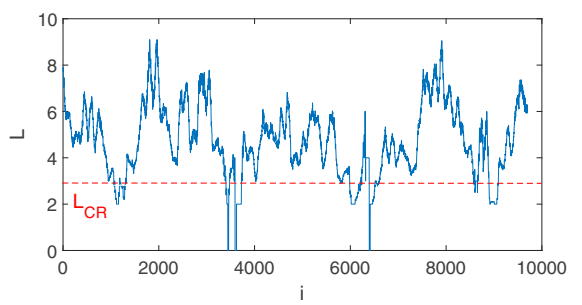

(k)

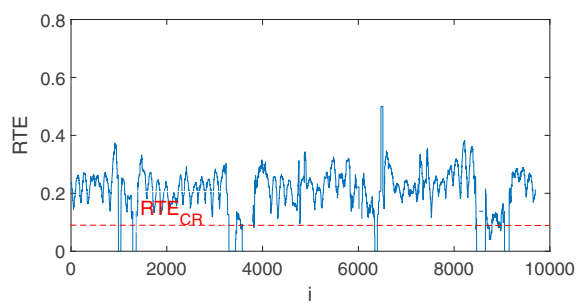

(b)

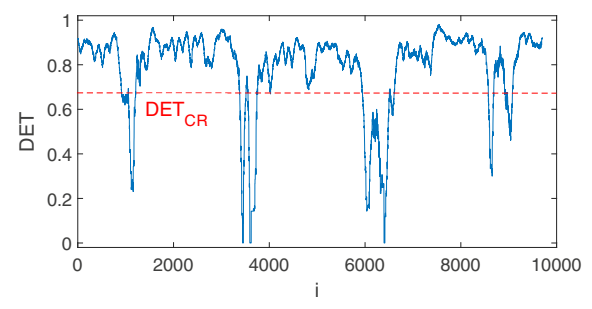

(d)

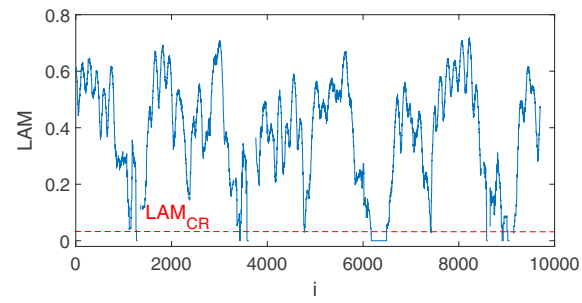

(f)

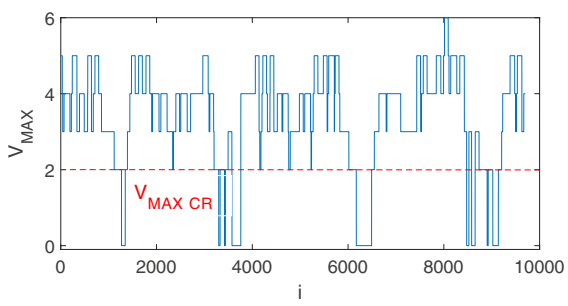

(h)

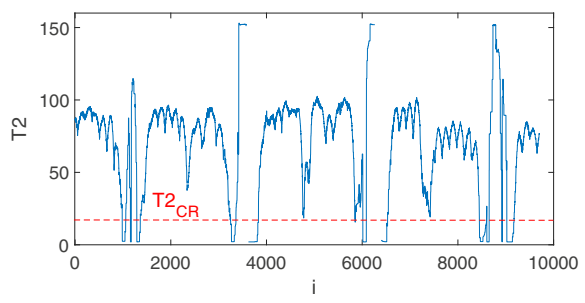

(j)

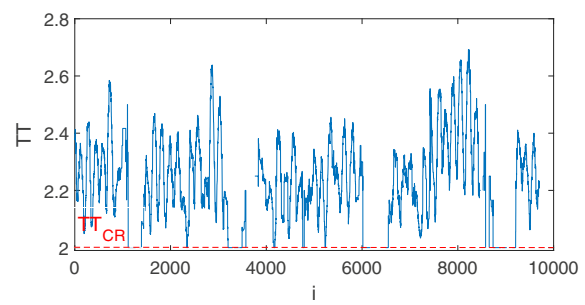

(1)

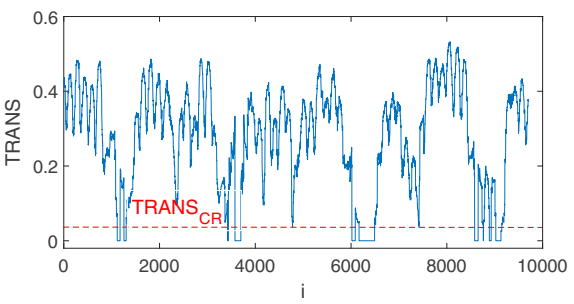


Fig. 7 Statistical results of RQA analysis obtained from recurrence diagram in Fig. 5b. The embedding parameters are $m=5$ and $d=17$, and the windowing sliding technique parameters were $\delta=300$, $\delta i=1$. The damage diameter was a constant of $2 \mathrm{~mm}$.

Changes in the RQA values can be used as the damage indicators (a)

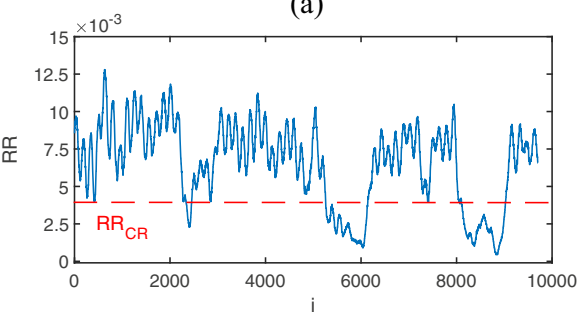

(c)

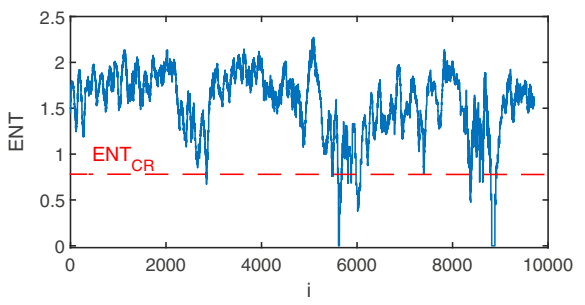

(e)

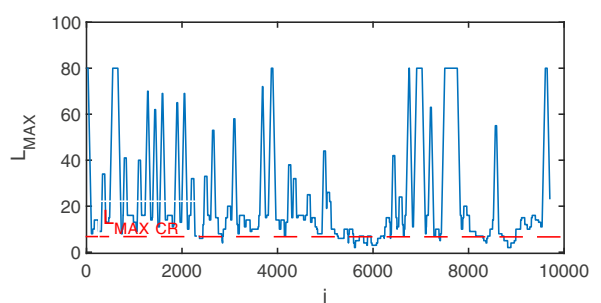

(g)

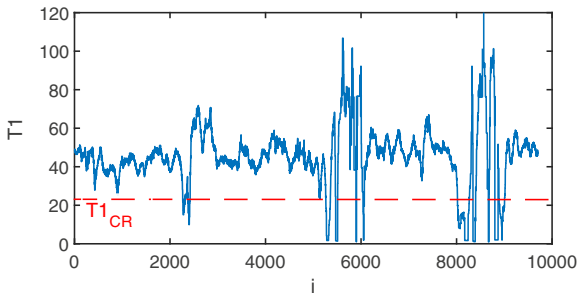

(i)

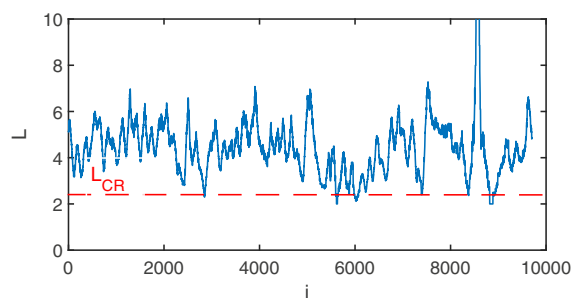

(k)

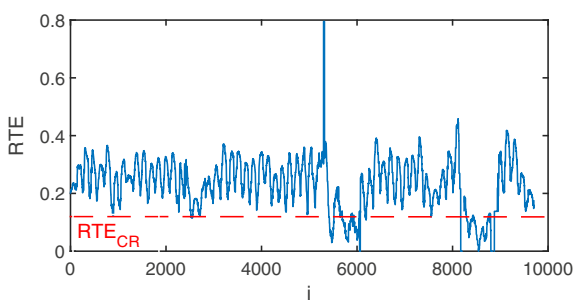

(b)

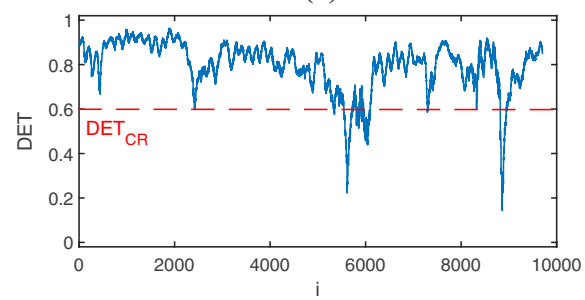

(d)

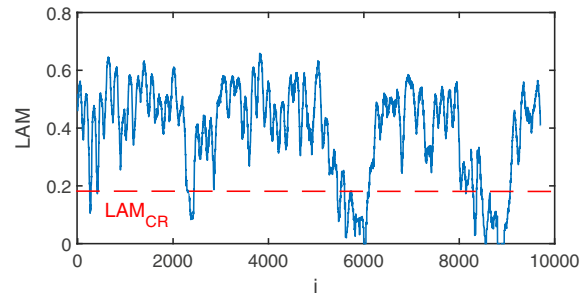

(f)

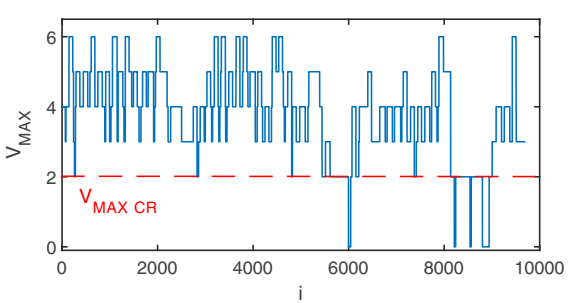

(h)

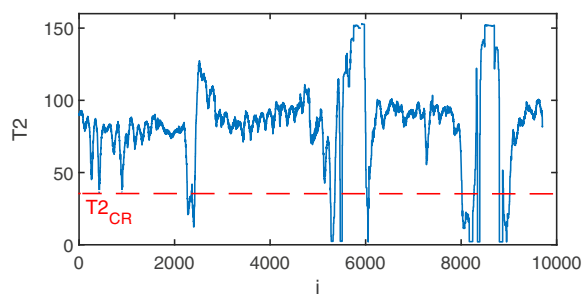

(j)

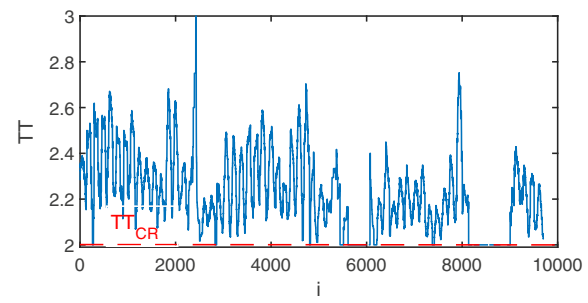

(1)

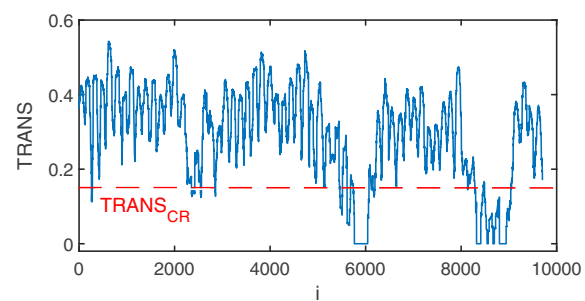


Table 2 Suggested critical and optimal recurrence quantifications for damage detection

\begin{tabular}{llll}
\hline RQA & Critical value (diameter) & Critical value (depth) & Optimal value \\
\hline$R R$ & 0.004 & 0.004 & 0.004 \\
$D E T$ & 0.694 & 0.600 & 0.628 \\
$E N T$ & 0.902 & 0.759 & 0.802 \\
$L A M$ & 0.037 & 0.173 & 0.132 \\
$T 1$ & 17.58 & 23.30 & 21.584 \\
$T 2$ & 15.64 & 37.90 & 31.222 \\
$R T E$ & 0.081 & 0.118 & 0.107 \\
$T R A N S$ & 0.038 & 0.144 & 0.112 \\
\hline
\end{tabular}

(a)

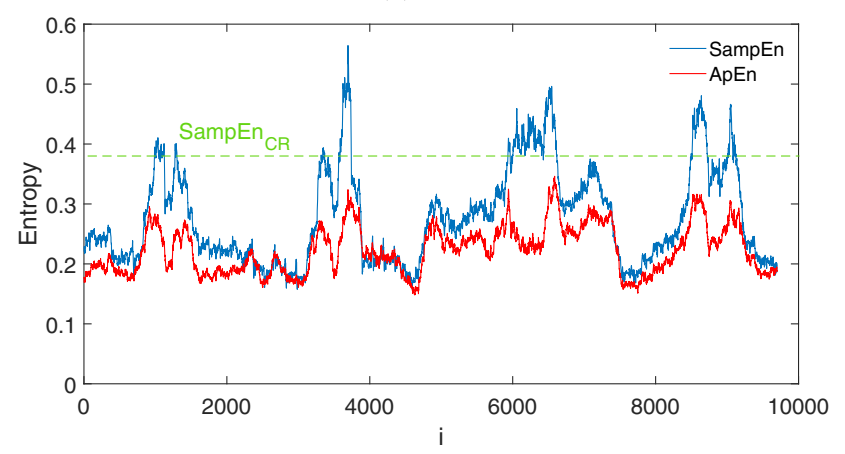

(b)

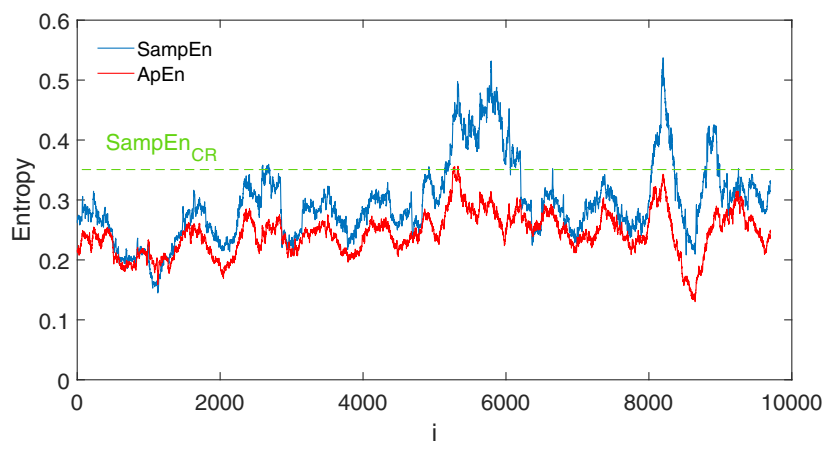

Fig. 8 Approximate and sample entropy values computed from the cutting force signal including different damage diameters (Fig. 4a) (a) and different damage depths (Fig. 4b)(b). The SampEn is capable of detecting defects

(a)

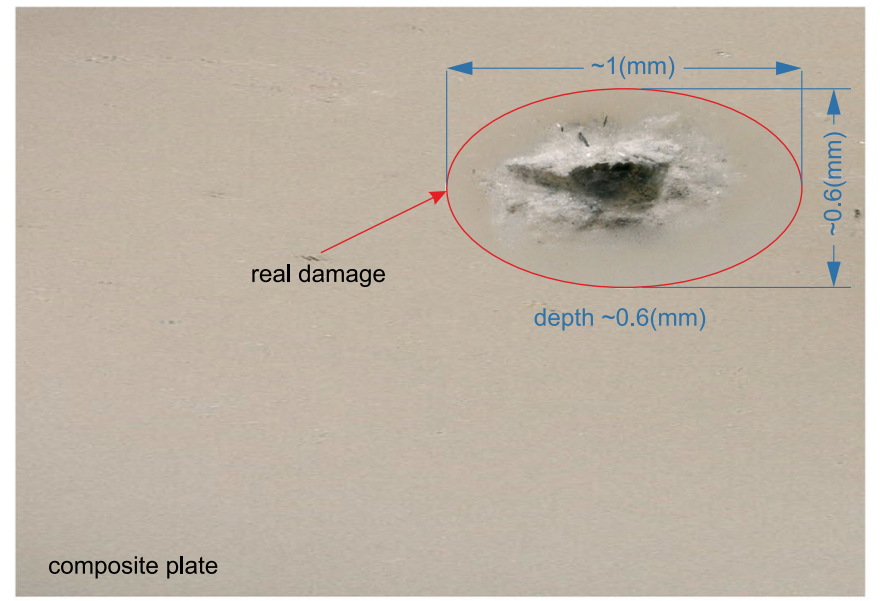

(b)

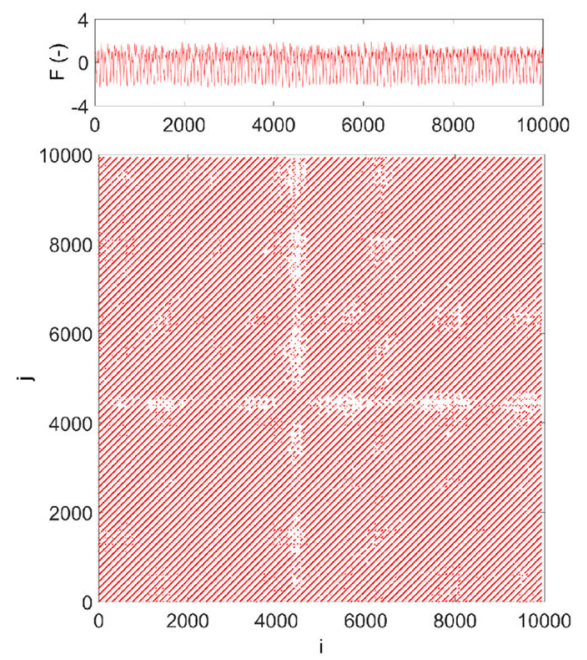

Fig. 9 Real impact damage of GFRP material (a) and the recurrence diagram obtained from the milling time series (b) 
(a)

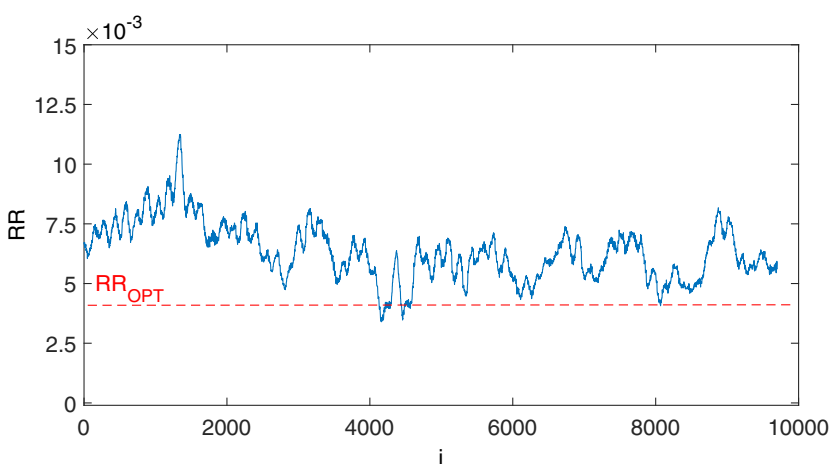

(c)

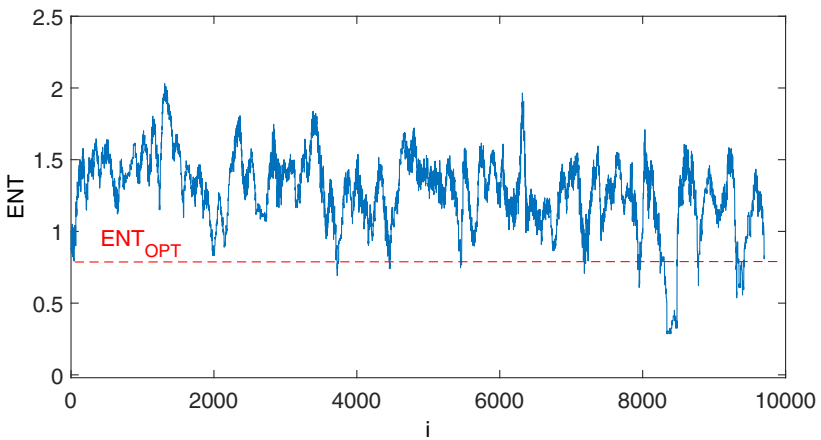

(e)

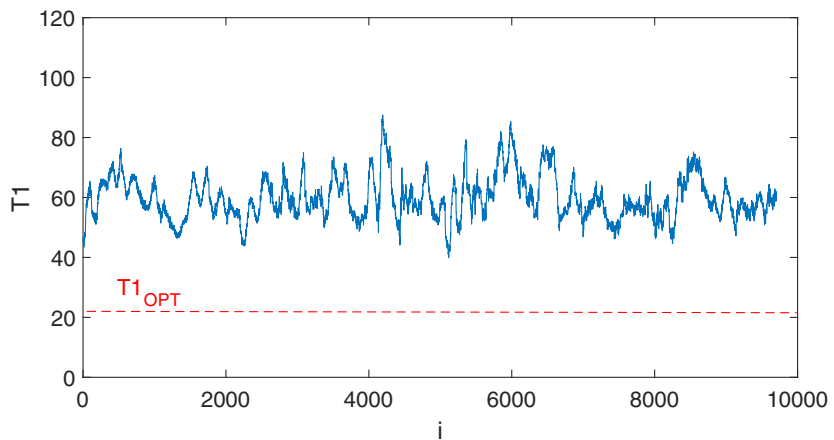

(g)

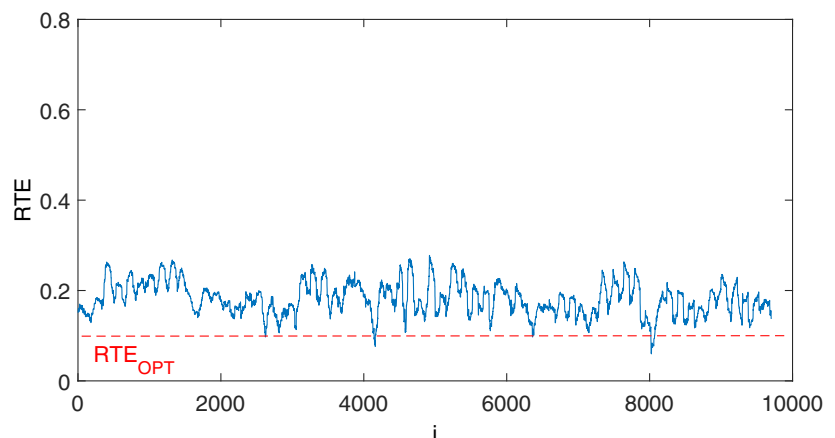

(b)

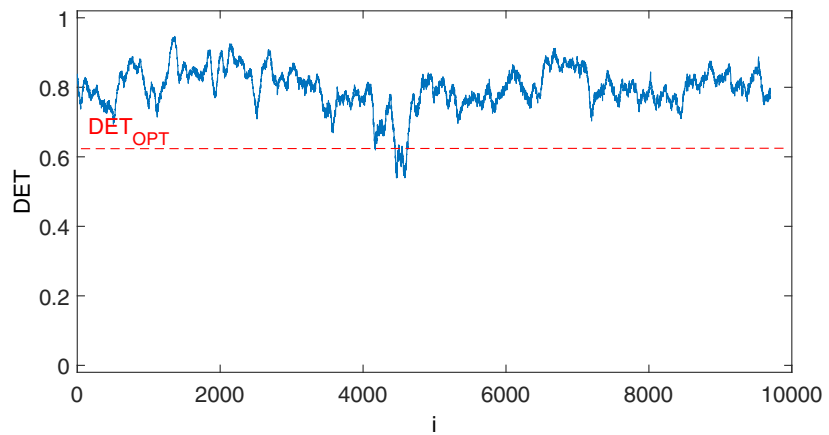

(d)

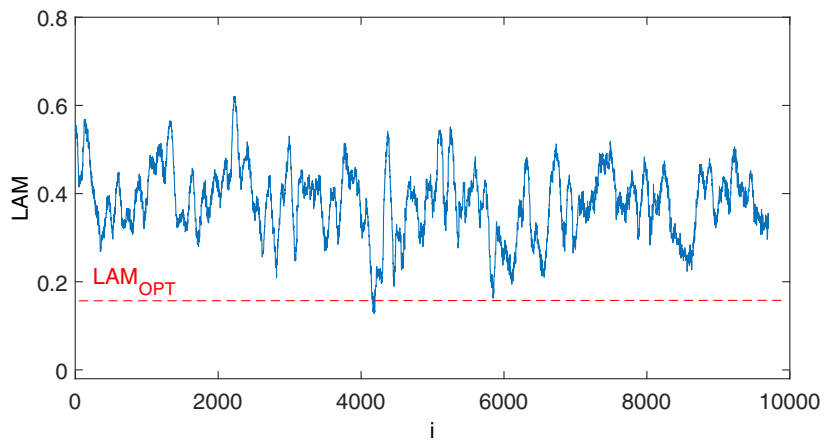

(f)

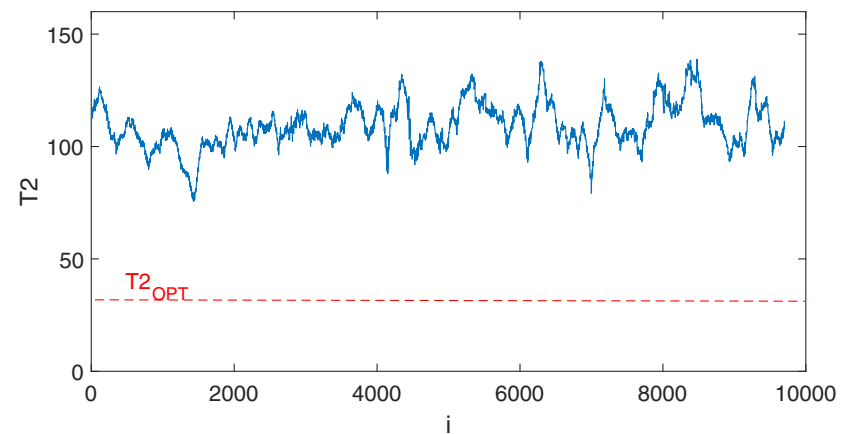

(h)

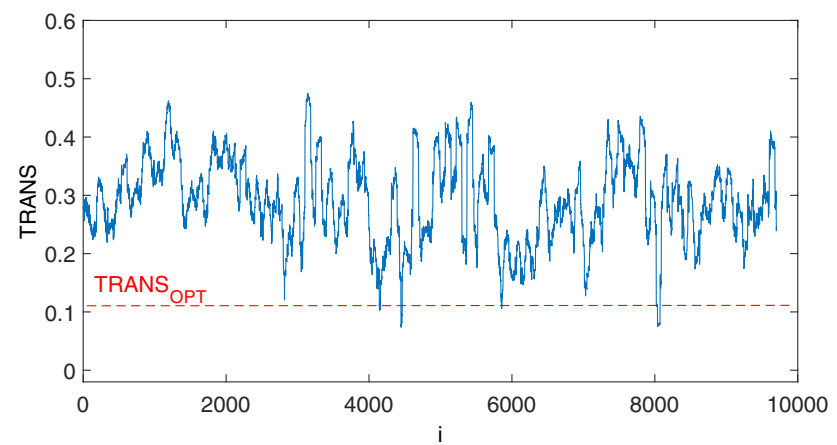

Fig. 10 Selected statistical results of RQA analysis of real damage detection: RR (a), DET (b), ENT (c), LAM (d), T1 (e), T2 (f), RTE (g), and TRANS (h). The embedding parameters are $m=5$ and $d=17$, and window moving technique parameters: $\delta=300, \delta i=1$ 
analysis, the optimal value of the SampEn is 0.36. Unfortunately, the ApEn cannot be used for damage detection in both cases (especially in the test no. 2).

\subsection{Real damage detection}

In order to check the proposed methods, an experiment milling test of the GFRP material with the real damage has been performed. The photo of the damage in Fig. 9a is shown. The macroscopic damage probably appeared as a result of impact of an object, caused one of the most common in-service defects, and had approximately diameter about $1 \mathrm{~mm} \times 0.6 \mathrm{~mm}$ and depth $0.6 \mathrm{~mm}$. The milling test was performed with the same parameters as the "hole" tests.

As we can see at the normalized cutting force, the damage is not observed (see Fig. 9b). Therefore, to detect damage, RP, RQA, and entropy analyses have been applied. The RP from the milling force with the real damage in Fig. $9 \mathrm{~b}$ is shown. Upon analyzing, we can find the unclear (empty) structure about $i=4300-4600$ data points. It is probably caused by a disturbance in the signal that is not visible in the course of the milling force. The RQA analysis was performed using only the proposed recurrence indicators in the previous section. The RQA results in Fig. 10 are shown. The red line means the optimal value indicator level.

Upon analyzing all recurrence indicators, we can say that detection of the real damage is more difficult because its shape is more complicated. In this case, the damage is relatively shallow; therefore, only recurrence indicators $R R$, $D E T$, and $L A M$ can detect it. Other indicators rather do not show changes in their value in relation to the average value. Note, that also the Shannon entropy (ENT) (Fig. 10c) disappoints. Therefore, the analysis of other entropy seems to be even more interesting.

The ApEn and SempEn entropy results in Fig. 11 are shown. The highest SampEn peaks correspond to the

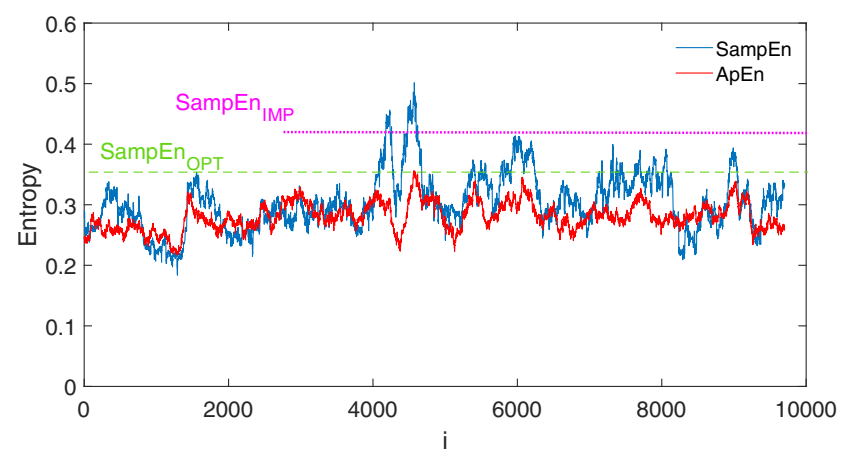

Fig. 11 SampEn and ApEn entropy calculated from the signal with the real damage. The parameters of entropy analysis are the same as in Section 4.3 damage position. The optimal value $\operatorname{SampEn}_{O P T}$ is a bit too low (green line); therefore, the optimal improved value $\operatorname{SampEn}_{I M P}$ (the pink line in Fig. 11) of 0.41 is proposed as the damage indicator. Again, the ApEn entropy course is on the similar level; no significant differences are observable (as in earlier analyses). Upon comparing both entropy, we can clearly confirm that only the SampEn as the damage indicator can be applied.

Measures derived from the RP, RQA, and entropy analyses have been shown to possess sensitivity to changing dynamics. Therefore, these methods can be used for the damage detection.

\section{Conclusion and summary}

In this paper, the different damage detection methods, namely the recurrence plot, the recurrence quantification, the approximate entropy, and the sample entropy, are proposed for damage detection basins of the milling time series. The obtained results in this work are a significant development and complement of the research presented in paper [31]. Accordingly, it has demonstrated more analyses including influence of damage depth and entropy analysis. Moreover, the milling tests on the composite material with the real damage have been performed.

The damage was modelled as the hole with the different diameters and depths. This allowed estimating the size of detectable defect. It has been shown that the proposed method is sensitive enough to determine the damage location, especially if the damage is significant in size (at least $4-5 \%$ of the cutter cross section). The obtained results show that the damage depth is crucial for detection. The damage with significant depth and different diameters is generally easy to detect by most recurrence quantifications. As a result, the optimal values of recurrence quantification are proposed. It is shown that the best recurrence qualifications are $R R, D E T$, and $L A M$. These selected recurrence quantifications agree with results in [27]. Additionally, the SampEn entropy can be also used as a damage indicator. Its optimal improved value is at the level of 0.41. Note, in the case of SampEn entropy, the optimal value should be improved about $15 \%$. The experiment with the normal life damage confirmed that the proposed recurrence indicators and SampEn are well and can be used in practice.

The proposed method has been proven to work well for structural health monitoring of composite structures and can be implemented directly during milling the process (needs a short time series). Moreover, it can also be applied to other machining processes.

There are few questions, however, which need to be solved in future works: damage located inside the material 
(invisible) or developing more detailed optimal values of recurrence quantification and entropy level. These problems will be analyzed in the next steps.

Funding The project/research was financed in the framework of the project Lublin University of Technology - Regional Excellence Initiative, funded by the Polish Ministry of Science and Higher Education (contract no. 030/RID/2018/19).

Open Access This article is licensed under a Creative Commons Attribution 4.0 International License, which permits use, sharing, adaptation, distribution and reproduction in any medium or format, as long as you give appropriate credit to the original author(s) and the source, provide a link to the Creative Commons licence, and indicate if changes were made. The images or other third party material in this article are included in the article's Creative Commons licence, unless indicated otherwise in a credit line to the material. If material is not included in the article's Creative Commons licence and your intended use is not permitted by statutory regulation or exceeds the permitted use, you will need to obtain permission directly from the copyright holder. To view a copy of this licence, visit http:// creativecommonshorg/licenses/by/4.0/.

\section{References}

1. Wojciechowski S, Matuszak M, Powalka B, Madajewski M, Marudad RW, Krolczyk GM (2019) Prediction of cutting forces during micro end milling considering chip thickness accumulation. Int J Mach Tools Manuf 147(103466):1-26

2. Wojciechowski S, Wiackiewicz M, Krolczyk GM (2018) Study on metrological relations between instant tool displacements and surface roughness during precise ball end milling. Measurement 129:686-694

3. Zhang J, Zhang S, Jiang D, Wang J, Lu S (2020) Surface topography model with considering corner radius and diameter of ball-nose end miller. Int J Adv Manuf Tech 106:3975-3984

4. Grossi N, Scippa A, Sallese L, Montevecchi F, Campatelli G (2018) On the generation of chatter marks in peripheral milling: a spectral interpretation. Int J Mach Tools Manuf 133:31-46

5. Smith RA (2009) Composite defects and their detection, materials science and engineering, vol. III- Composite Defects and Their Detection. EOLSS, Paris, France

6. Sem JK, Everett RA (2000) RTO/NATO, ISBN 92-837-1051-7, 5.1-5.21

7. Ashir M, Nocke A, Cherif Ch (2019) Effect of the position of defined local defect on the mechanical performance of carbonfiber-reinforced plastics. Autex Res J 19(1):74-79

8. Barry TJ, Kesharaju M, Nagarajah C (2016) Defect characterisation in laminar composite structures using ultrasonic techniques and artificial neural networks. J Compos Mater 50:861-871

9. Smith RA, Jones LD, Willsher SJ, Marriott AB (1998) Diffraction and shadowing errors in - $6 \mathrm{~dB}$ defect sizing of delaminations in composites. Brit J Nondestr Test 40(1):44-49

10. Smith RA, Clarke B (1994) Ultrasonic C-scan determination of ply stacking sequence in carbonfiber composites. Brit J Nondestr Test 36(10):741-747

11. Balasko M, Svab E, Molnar G, Veres I (2005) Classification of deffects in honeycomb composite structure of helicopter rotor blades. Nucl Instrum Methods Phys Res A 542:45-51

12. Cawley $P$ (1987) The sensitivity of the mechanical impedance method of non-destructive testing. NDT Int 20:209-215
13. Kriechenbauer S, Mauermann R, Muller P (2014) Deep drawing with superimposed low-frequency vibrations on servo-screw presses. Procedia Eng 81:905-913

14. Meng DA, Zhao X, Li J, Zhao S, Han Q (2017) Mechanical behavior and microstructure of low-carbon steel undergoing lowfrequency vibration-assisted tensile deformation. J Mater Res 32:3885-3893

15. Takens F (1981) Detecting strange attractors in turbulence. In: Rand DA, Young LS (eds) Dynamical systems and turbulence. Springer, Berlin, pp 366-381

16. Packard NH, Crutschfield JP, Farmer JD, Shaw RS (1980) Geometry from a time series. Phys Rev Lett 45:712-716

17. Grassberger P, Procaccia I (1983) Characterization of strange attractors. Phys Rev Lett 50:346-349

18. Kantz H (1994) A robust method to estimate the maximal Lyapunov exponent of a time series. Phys Rev Lett 185:77-87

19. Farmer JD (1982) Information dimension and the probabilistic structure of chaos. Z Naturforsch 37:1304-1326

20. Sohn H, Farrar CHR (2001) Damage diagnosis using time series analysis of vibration signals. Smart Mater Struct 10:1-6

21. Packard NH, Crutschfield JP, Farmer JD, Shaw RS (2019) A brief introduction to nonlinear time series analysis and recurrence plots. Vibration 2:332-368

22. Bai A, Hira S, Parag DS (2017) Recurrence based similarity identification of climate data. Discrete Dyn Nat Soc ID 7836720:1-21

23. Litak G, Syta A, Rusinek R (2011) Dynamical changes during composite milling: recurrence and multiscale entropy analysis. Int J Adv Manuf Tech 56:445-453

24. Kecik K, Rusinek R, Warminski J (2011) Stability lobes analysis of nickel superalloys milling. Int J Bifurcat Chaos 21(1):29432954

25. Kecik K, Borowiec M, Rusinek R (2016) Verification of the stability lobes of Inconel 718 milling by recurrence plot applications and composite multiscale entropy analysis. Eur Phys J Plus 131(14):1-9

26. Iwaniec J, Uhl T, Staszewski WJ, Klepka A (2012) Etection of changes in cracked aluminium plate determinism by recurrence analysis. Nonlinear Dyn 70:125-140

27. Nichols JM, Trickey ST, Seaver M (2006) Damage detection using multivariate recurrence quantification analysis. Mech Syst Signal Process 20:421-437

28. Qian Y, Yan R, Hu S (2014) Bearing degradation evaluation using recurrence quantification analysis and Kalman filter. IEEE Trans Instrum Meas 63:2599-2610

29. Hou Y, Aldrich C, Lepkova K, Machuca LL, Kinsella B (2016) Monitoring of carbon steel corrosion by use of electrochemical noise and recurrence quantification analysis. Corros Sci 112:6372

30. Yang Y, Zhang T, Shao Y, Meng G (2010) Effect of hydrostatic pressure on the corrosion behaviour of Ni-Cr-Mo-V high strength steel. Corros Sci 52:2697-2706

31. Kecik K, Ciecielag K, Zaleski K (2017) Damage detection of composite milling process by recurrence plots and quantifications analysis. Int J Adv Manuf Tech 89:133-144

32. Mhalsekar SD, Shrikantha MG, Rao S, Gangadharan KV (2009) Determination of transient and steady state cutting in face milling operation using recurrence quantification analysis. ARPN J Eng Appl Sci 4(10):36-46

33. Fraser AM, Swinney HL (1986) Independent coordinates for strange attractors from mutual information. Phys Rev A 33:11341140

34. Kennel M, Brown R, Abarbanel H (1992) Determining embedding dimension for phase space reconstruction using a geometrical construction. Phys Rev A 45:3403-3411 
35. Eckmann JP, Kamphorst SO, Ruelle D (1987) Recurrence plots of dynamical system. Europhys Lett 4:973-977

36. Zbilut JP, Webber CL (1992) Embeddings and delays as derived from quantification of recurrence plots. Phys Lett A 171:199-203

37. Webber CL Jr., Zbilut JP (1994) Dynamical assessment of physiological systems and states using recurrence plot strategies. J Appl Physiol 76(2):965-973

38. Marwan N, Romano MC, Thiel M, Kurths J (2007) Recurrence plots for the analysis of complex systems. Phys Rep 438:237-329

39. Schinkel S, Dimigen O, Marwan N (2008) Selection of recurrence threshold for signal detection. Eur Phys J Spec Top 164:45-53

40. Marwan N, Wessel N, Meyerfeldt U, Schirdewan A, Kurths J (2002) Recurrence-plot-based measures of complexity and their application to heart-rate-variability data. Phys Rev E 026702:66

41. Gao J, Cai H (2000) On the structures and quantification of recurrence plots. Phys Lett A 270:75-87

42. Marwan N, Donges JF, Zou Y, Donner RV, Kurths J (2009) Complex network approach for recurrence analysis of time series. Phys Lett A 373(46):4246-4254

43. Benish WA (2020) A review of the application of information theory to clinical diagnostic testing. Entropy 22(97):1-20

44. Borkowska M (2016) Entropy-based algorithms in the analysis of biomedical signals. Studies Log Gramm Rhetor 43(1):21-32

45. Kaffashi F, Foglyano R, Wilson ChG, Loparo KA (2008) The effect of time delay on approximate \& sample entropy calculations. Physica D 237:3069-3074

46. Pincus SM (1991) Approximate entropy as a measure of system complexity. Proc Natl Acad Sci U S A 88(6):2297-2301
47. Chen W, Zhuang J, Yu W, Wang Z (2009) Measuring complexity using FuzzyEn, ApEn, and SampEn. Med Eng Phys 31:61-68

48. Li J, Cai J, Peng Y, Zhang X, Zhou C, Li G, Tang J (2019) Magnetotelluric signal-noise identification and separation based on apen-mse and stomp. Entropy 21:1-15

49. Richman JS, Moorman JR (2000) Physiological time-series analysis using approximate entropy and sample entropy. Am J Physiol Heart Circ Physiol 278:2039-2049

50. https://tocsy.pik-potsdam.de/CRPtoolbox/

51. Webber CHL Jr., Ioana C, Marwan N (2016) Recurrence plots and their quantications: expanding horizons. Springer International Publishing

52. Ciecielag K (2019) Influence of milling conditions on the geometric structure of the surface of the selected polymer composites. PhD Thesis, Lublin University of Technology

53. Ciecielag K, Kecik K, Zaleski K (2020) Effect of depth surface defects in carbon fibre reinforced composite material on the selected recurrence quantifications. Adv Mater Sci 20(2):71-80

54. Ciecielag K, Kecik K, Zaleski K (2020) Defects detection from time series of cutting force in composite milling process by recurrence analysis. J Reinf Plast Compos 6:1-12

55. Ciecielag K, Kecik K, Zaleski K (2017) Influence of defect diameter on its detection in milling process of composite material using recurrence plot technique. Compos Theory and Pract 4:194199

Publisher's note Springer Nature remains neutral with regard to jurisdictional claims in published maps and institutional affiliations. 